\section{(6) OPEN ACCESS}

\title{
Non-major bleeding with apixaban versus warfarin in patients with atrial fibrillation
}

\author{
M Cecilia Bahit, ${ }^{1}$ Renato D Lopes, ${ }^{2}$ Daniel M Wojdyla ${ }_{1}{ }^{2}$ Claes Held, ${ }^{3}$ Michael Hanna, ${ }^{4}$ \\ Dragos Vinereanu, ${ }^{5}$ Elaine M Hylek, ${ }^{6}$ Freek Verheugt, ${ }^{7}$ Shinya Goto, ${ }^{8}$ \\ John H Alexander, ${ }^{2}$ Lars Wallentin, ${ }^{3}$ Christopher B Granger ${ }^{2}$
}

'INECO Neurociencias Oroño, Rosario, Santa Fe, Argentina ${ }^{2}$ Duke Clinical Research Institute, Duke University School of Medicine, Durham, North Carolina, USA

3Uppsala Clinical Research Center, Uppsala University, Uppsala, Sweden

${ }^{4}$ Bristol-Myers Squibb, Princeton, New Jersey, USA

${ }^{5}$ University of Medicine and Pharmacy Carol Davila,

Bucharest, Romania

${ }^{6}$ Boston University Medical Center, Boston, Massachusetts, USA

${ }^{7}$ Heartcenter, Onze Lieve Vrouwe Gasthuis (OLVG), Amsterdam, Netherlands

${ }^{8}$ Tokai University School of Medicine, Isehara, Japan

\section{Correspondence to} Dr Renato D Lopes, Duke Clinical Research Institute Room 0311 Terrace Level, 2400 Pratt Street, Durham, NC 27705, USA; renato.lopes@duke.edu

Received 28 April 2016 Revised 22 September 2016 Accepted 27 September 2016 Published Online First 24 October 2016

\section{ABSTRACT}

Objective We describe the incidence, location and management of non-major bleeding, and assess the association between non-major bleeding and clinical outcomes in patients with atrial fibrillation (AF) receiving anticoagulation therapy enrolled in Apixaban for Reduction in Stroke and other Thromboembolic Events in Atrial Fibrillation (ARISTOTLE).

Methods We included patients who received $\geq 1$ dose of study drug $(n=18$ 140). Non-major bleeding was defined as the first bleeding event considered to be clinically relevant non-major (CRNM) or minor bleeding, and not preceded by a major bleeding event.

Results Non-major bleeding was three times more common than major bleeding ( $12.1 \%$ vs $3.8 \%)$. Like major bleeding, non-major bleeding was less frequent with apixaban (6.4 per 100 patient-years) than warfarin (9.4 per 100 patient-years) (adjusted HR 0.69, 95\% Cl 0.63 to 0.75 ). The most frequent sites of non-major bleeding were haematuria (16.4\%), epistaxis (14.8\%), gastrointestinal $(13.3 \%)$, haematoma $(11.5 \%)$ and bruising/ecchymosis (10.1\%). Medical or surgical intervention was similar among patients with non-major bleeding on warfarin versus apixaban (24.7\% vs $24.5 \%$ ). A change in antithrombotic therapy (58.6\% vs $50.0 \%$ ) and permanent study drug discontinuation $(5.1 \%$ (61) vs $3.6 \%(30), p=0.10)$ was numerically higher with warfarin than apixaban. CRNM bleeding was independently associated with an increased risk of overall death (adjusted HR 1.70, 95\% Cl 1.32 to 2.18) and subsequent major bleeding (adjusted HR 2.18, 95\% Cl 1.56 to 3.04).

Conclusions In ARISTOTLE, non-major bleeding was common and substantially less frequent with apixaban than with warfarin. CRNM bleeding was independently associated with a higher risk of death and subsequent major bleeding. Our results highlight the importance of any severity of bleeding in patients with AF treated with anticoagulation therapy and suggest that non-major bleeding, including minor bleeding, might not be minor. Trial registration number NCT00412984; post-results.

Atrial fibrillation (AF) is an important risk factor for stroke. Anticoagulation is highly effective in reducing thromboembolic events; however, bleeding complications are common in this setting. Although warfarin reduces the risk of stroke in patients with AF by $64 \%,{ }^{1}$ there is a risk of bleeding that may relate in part to its narrow therapeutic window and difficulties in adjusting dosing. ${ }^{2}$
Non-vitamin K oral anticoagulants (NOACs) are effective alternatives to warfarin due to their proven efficacy in reducing stroke and thromboembolic events and safer profile. ${ }^{3-5}$ In the Apixaban for Reduction in Stroke and other Thromboembolic Events in Atrial Fibrillation (ARISTOTLE) trial, ${ }^{3}$ apixaban compared with warfarin significantly reduced the risk of stroke, major bleeding, intracranial haemorrhage and death in patients with $\mathrm{AF}^{6}$ In addition, it has been found that major bleeding was associated with substantially increased risk of death, ischaemic stroke or myocardial infarction, especially following intracranial haemorrhage.

However, the relevance and importance of preventing non-major bleeding are less clear. In the present study, we sought to characterise non-major bleeding events and their management, and to evaluate the association between non-major bleeding and subsequent clinical outcomes.

\section{METHODS}

The ARISTOTLE trial design and results have been reported previously. ${ }^{3} 8$ Patients with AF and at least one risk factor for stroke were randomised to receive either dose-adjusted warfarin (international normalised ratio (INR) 2-3) or apixaban $5 \mathrm{mg}$ twice daily. A reduced dose of apixaban, $2.5 \mathrm{mg}$ twice daily, was designated for participants with two or more of the following criteria: age $\geq 80$ years, weight $\leq 60 \mathrm{~kg}$ or serum creatinine concentration $\geq 1.5 \mathrm{mg} / \mathrm{dL}(133 \mathrm{mmol} / \mathrm{L})$. In the overall trial population, the reduced dose of apixaban (or placebo) was administered to 831 patients (4.7\%). To enhance the quality of warfarin management, a dosage algorithm was provided and regular feedback was provided to sites regarding their level of INR control. Specifically, patients were excluded with conditions other than AF that required anticoagulation (eg, a prosthetic heart valve), stroke within the previous 7 days, a need for aspirin at a dose $>165 \mathrm{mg}$ a day, or a need for both aspirin and clopidogrel. Approval by the appropriate ethics committees was obtained at all sites. All patients provided written informed consent.

\section{Study population}

For the purpose of this study, we included all patients receiving at least one dose of study drug. Three groups of patients were identified: patients with no bleeding, patients in whom the first
To cite: Bahit MC, Lopes RD, Wojdyla DM, et al. Heart 2017:103:623-628. 
bleeding event was clinically relevant non-major or minor and patients in whom the first bleeding event was major. The analyses of bleeding events included all events from the time of the first dose of study drug until 2 days after the last dose was received.

\section{Bleeding definitions}

Non-major bleeding was the composite of bleeding events considered to be (a) clinically relevant non-major bleeding or (b) minor bleeding. Major bleeding was defined according to International Society on Thrombosis and Haemostasis criteria as clinically overt bleeding accompanied by a decrease in the haemoglobin level of at least $2 \mathrm{~g} / \mathrm{dL}$ or requiring a transfusion of at least 2 units of packed red blood cells, occurring at a critical site (intracranial, intraocular, intraspinal, intra-articular, intramuscular with compartment syndrome, pericardial, retroperitoneal), or resulting in death. ${ }^{9}$

Clinically relevant non-major bleeding was defined as acute or subacute clinically overt bleeding that did not satisfy the criteria for major bleeding and led to hospital admission for bleeding, physician-guided medical or surgical treatment for bleeding, or a change in antithrombotic therapy (including study drug) due to bleeding. All acute clinically overt bleeding events not meeting the criteria for either major or clinically relevant nonmajor bleeding were classified as minor bleeding. Laboratory and transfusion data coupled with clinical event details were used to identify and adjudicate potential bleeding events. Routine collection of haemoglobin occurred every 3 months. Location of bleeding was extracted from the case report form. Additional source documents were collected when necessary. The primary safety outcomes were adjudicated on the basis of prespecified criteria by a clinical events committee whose members were not aware of study group assignments.

\section{Statistical analysis}

Baseline characteristics were presented for patients according to no bleeding, non-major bleeding and major bleeding. Continuous variables were summarised as medians and quartiles and categorical variables as frequencies and percentages. All-cause mortality and ischaemic stroke after the bleeding events were analysed using a Cox proportional model with separate time-dependent variables for bleeding (intracranial, major non-intracranial, clinically relevant non-major and minor). The following variables were included in the adjusted results: type of AF; age; sex; region; history of myocardial infarction; congestive heart failure; stroke, transient ischaemic attack, or systemic embolism; diabetes; time with AF; hypertension; history of bleeding; prior vitamin $\mathrm{K}$ antagonist (VKA) use; $\mathrm{CHADS}_{2}$ score; history of vascular disease; creatinine clearance; angiotensin converting enzyme inhibitors, angiotensin II receptor blockers, or amiodarone at randomisation; and randomised treatment. Kaplan-Meier curves were used to summarise the incidence of non-major bleeding by randomised treatment. All analyses were performed with SAS V.9.4 (SAS Institute, Cary, North Carolina, USA).

\section{RESULTS}

Non-major bleeding was three times more common than major bleeding (12.1\% (2204) vs 3.8\% (692)). Non-major bleeding was less frequent with apixaban (6.4 per 100 patient-years) than warfarin (9.4 per 100 patient-years) (HR (apixaban vs warfarin) $0.69,95 \%$ CI 0.63 to 0.75 ) (figure 1 ).

\section{Baseline characteristics}

Baseline characteristics for patients with no bleeding, non-major bleeding and major bleeding are shown in table 1 .

Patients with non-major bleeding had higher risk features compared with patients with no bleeding, but lower than those who suffered major bleeding first. Mean $\mathrm{CHA}_{2} \mathrm{DS}_{2}$-VASc scores (standard deviation) for patients with no bleeding, non-major bleeding and major bleeding were 3.4 (1.5), 3.6 (1.5) and 3.8 (1.5), respectively. Renal dysfunction (mild and moderate impairment) was more common with more severe bleeding. Use of aspirin was similar in the three groups, but clopidogrel and non-steroidal anti-inflammatory agent use was higher in those with non-major and major bleeding compared with those with no bleeding. History of anaemia was almost twice as frequent in patients with non-major and major bleeding.

\section{Location of haemorrhage}

Overall, the most frequent sites of non-major bleeding were haematuria (16.4\%), epistaxis $(14.8 \%)$, gastrointestinal bleeding (13.3\%), haematoma (11.5\%) and bruising/ecchymosis $(10.1 \%)$
Figure 1 Cumulative incidence curves of first non-major bleeding event by treatment.

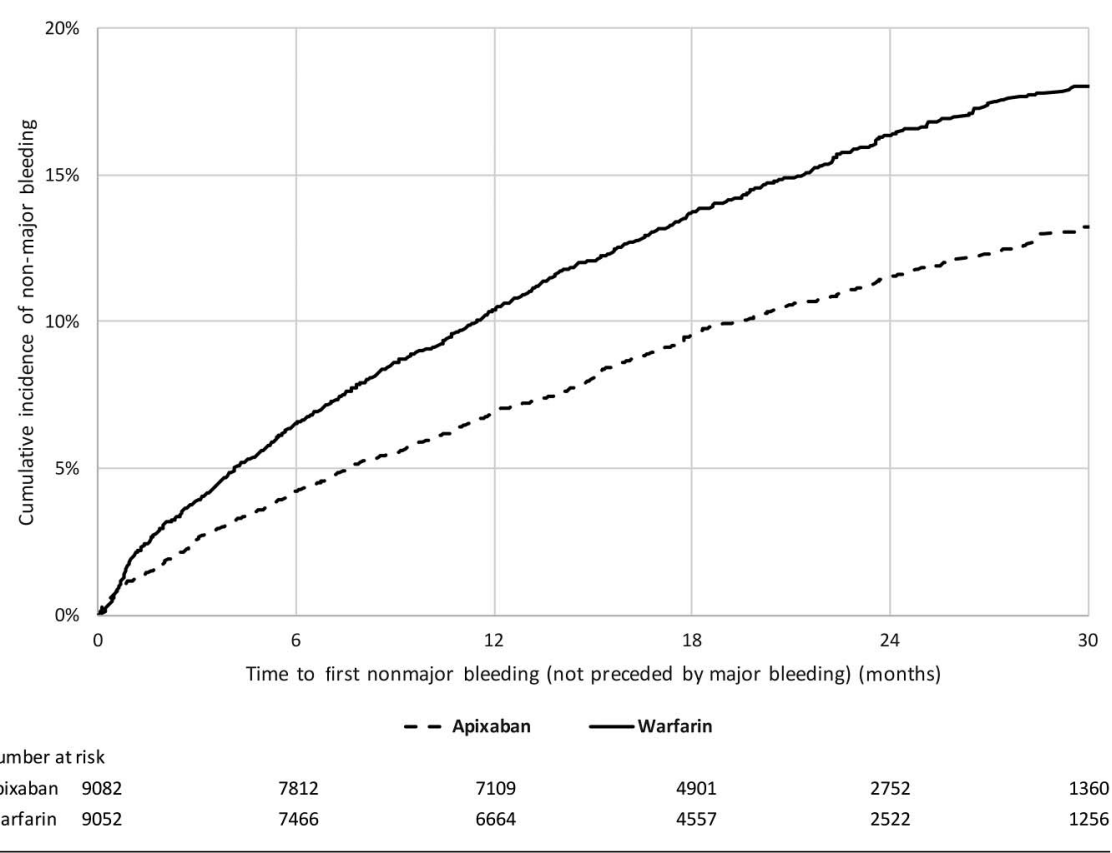

Bahit MC, et al. Heart 2017;103:623-628. doi:10.1136/heartjnl-2016-309901 


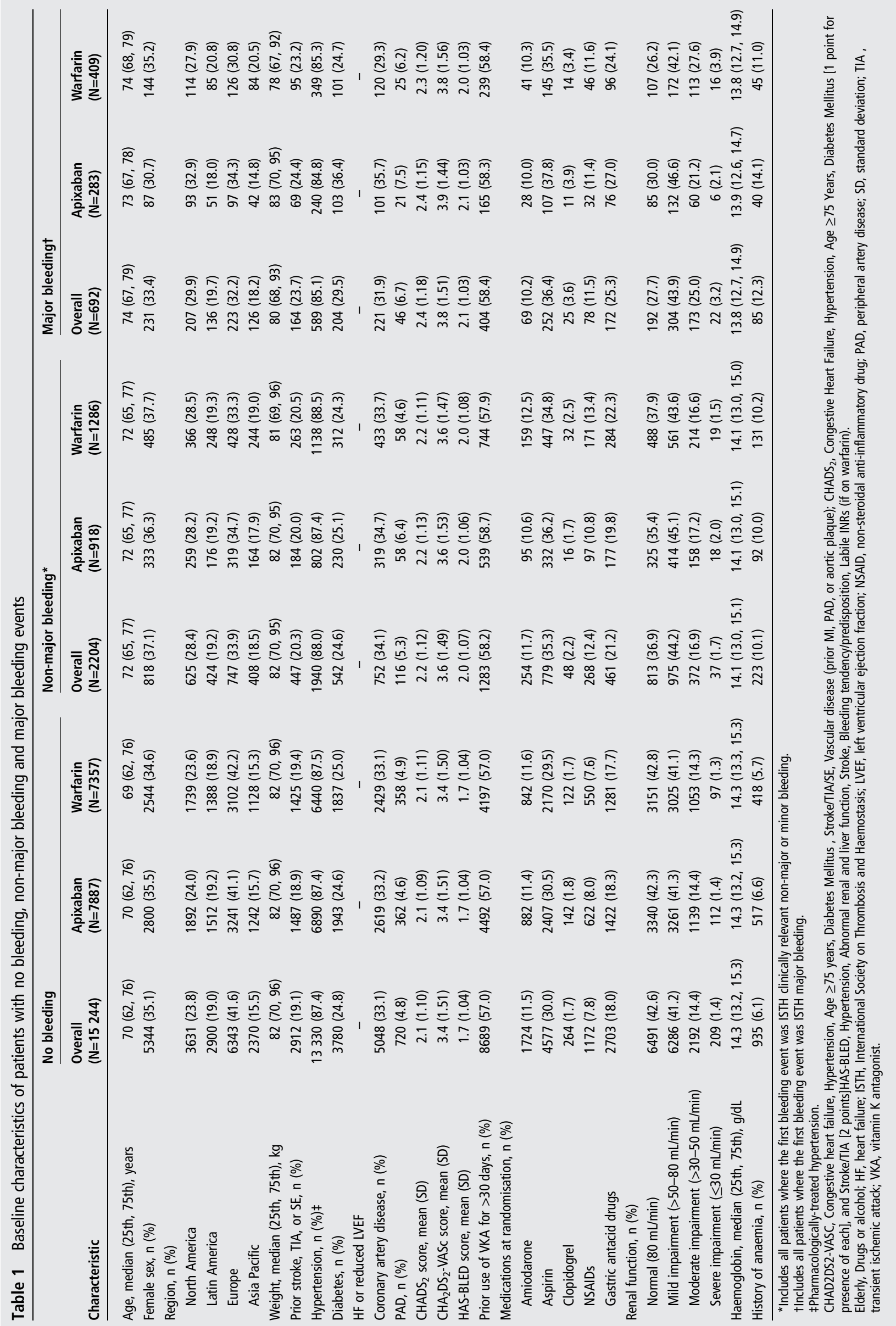


(figure 2A); these locations account for 69\% of total non-major bleeding. For each location, bleeding was numerically lower for apixaban compared with warfarin except for lower gastrointestinal bleeding (figure 2B). Lower gastrointestinal bleeding and haemorrhoidal bleeding appeared to be more common with apixaban compared with warfarin; however, upper gastrointestinal bleeding appeared to be more common with warfarin.

\section{Characteristics and circumstances of first non-major bleeding event}

Of the patients with non-major bleeding, in general, medical or surgical consultations and interventions were similar among those treated with apixaban versus warfarin (table 2).

Hospitalisations for non-major bleeding events were slightly more frequent in patients treated with warfarin versus apixaban (13.8\% (178) vs $12.9 \%(118))$. Of those who suffered nonmajor bleeding, change in antithrombotic therapy $(58.6 \%(754)$ vs $50.0 \%(459), \mathrm{p}<0.0001)$ and permanent study drug discontinuation $(5.1 \%(61)$ vs $3.6 \%(30), p=0.10)$ were also numerically higher with warfarin than apixaban.

\section{Association between different severity of bleeding and subsequent clinical outcomes}

Associations between different severity of bleeding and subsequent outcomes are shown in figure 3. Clinically relevant nonmajor bleeding was associated with an increased risk of overall death (adjusted HR 1.70, 95\% CI 1.32 to 2.18 ) to a lower extent than major non-intracranial bleeding (adjusted HR 2.34, 95\% CI 1.84 to 2.99 ), but to a higher extent than minor bleeding (adjusted HR 1.12, 95\% CI 0.90 to 1.40). Among patients with non-major bleeding, subsequent 30-day mortality was greater among patients who stopped (10.2\%) than those who continued (4.9\%) their anticoagulant study drug (HR 2.1, 95\% CI 1.4 to 3.1). There was also an association between different severities of bleeding and subsequent ischaemic stroke that did not reach statistical significance.

After multivariable adjustment, minor bleeding and clinically relevant non-major bleeding were associated with an increase in subsequent major bleeding (adjusted HR 1.53, 95\% CI 1.19 to 1.97 and adjusted HR 2.18, 95\% CI 1.56 to 3.04, respectively). There was a non-significant association between non-major bleeding and minor bleeding and intracranial haemorrhage (adjusted HR 1.68, 95\% CI 0.73 to 3.83 and adjusted HR 1.14, $95 \%$ CI 0.61 to 2.12 , respectively).

\section{DISCUSSION}

In the ARISTOTLE trial, non-major bleeding was more common than major bleeding and occurred less frequently with

Table 2 Characteristics and circumstances of the first clinically relevant non-major or minor bleeding event according by study drug

\begin{tabular}{|c|c|c|c|}
\hline & $\begin{array}{l}\text { Overall } \\
(n=2204)\end{array}$ & $\begin{array}{l}\text { Apixaban } \\
(\mathrm{n}=918)\end{array}$ & $\begin{array}{l}\text { Warfarin } \\
(n=1286)\end{array}$ \\
\hline \multicolumn{4}{|l|}{ Characteristics } \\
\hline Fall in haemoglobin $<2 \mathrm{~g} / \mathrm{dL}$ & $268(12.2)$ & $118(12.9)$ & $150(11.7)$ \\
\hline Clinically overt & $1012(45.9)$ & $430(46.8)$ & $582(45.3)$ \\
\hline Lead to transfusion & $40(1.8)$ & $13(1.4)$ & $27(2.1)$ \\
\hline $\begin{array}{l}\text { Required medical or surgical } \\
\text { consultation }\end{array}$ & $1624(73.7)$ & $723(78.8)$ & $901(70.1)$ \\
\hline $\begin{array}{l}\text { Required medical or surgical } \\
\text { intervention }\end{array}$ & $543(24.6)$ & 225 (24.5) & $318(24.7)$ \\
\hline $\begin{array}{l}\text { Caused change in antithrombotic } \\
\text { therapy }\end{array}$ & $1213(55.0)$ & $459(50.0)$ & $754(58.6)$ \\
\hline Led to hospitalisation & $296(13.4)$ & $118(12.9)$ & $178(13.8)$ \\
\hline \multicolumn{4}{|l|}{ Circumstances } \\
\hline Spontaneous & $1564(71.0)$ & $638(69.5)$ & $926(72.0)$ \\
\hline Trauma & $529(24.0)$ & $214(23.3)$ & $315(24.5)$ \\
\hline Procedure-related & $204(9.3)$ & $100(10.9)$ & $104(8.1)$ \\
\hline \multicolumn{4}{|l|}{ Discontinuations } \\
\hline Permanent & $91(4.4)$ & $30(3.6)$ & $61(5.1)$ \\
\hline Temporary & $527(25.7)$ & $219(25.9)$ & $308(25.5)$ \\
\hline
\end{tabular}

apixaban than warfarin. When non-major bleeding occurred, apixaban was less frequently discontinued than warfarin.

Minor bleeding was associated with higher risks of ischaemic stroke, death and subsequent major bleeding. Importantly, clinically relevant non-major bleeding was independently associated with a significant 2.18 -fold higher risk of major bleeding and 1.7-fold higher risk of death. The increased risk of major bleeding and death following clinically relevant non-major bleeding should be interpreted with caution when making decisions in clinical practice about treatment options because it was derived from a time-dependent analysis based on information collected during the study and not on routine clinical practice.

The observation that non-major bleeding was three times more common than major bleeding was similar to other AF trials testing new oral anticoagulants. In the Rivaroxaban once Daily Oral Direct Factor Xa Inhibition Compared with Vitamin K Antagonism for Prevention of Stroke and Embolism Trial in Atrial Fibrillation (ROCKET AF) trial, ${ }^{5}$ the primary safety endpoint included both major and clinically relevant non-major bleeding. Clinically relevant non-major bleeding accounted for most of the events, representing three out of four bleeding events. In ROCKET AF, $16.7 \%$ of the patients treated with

B Number of non-major bleeding events
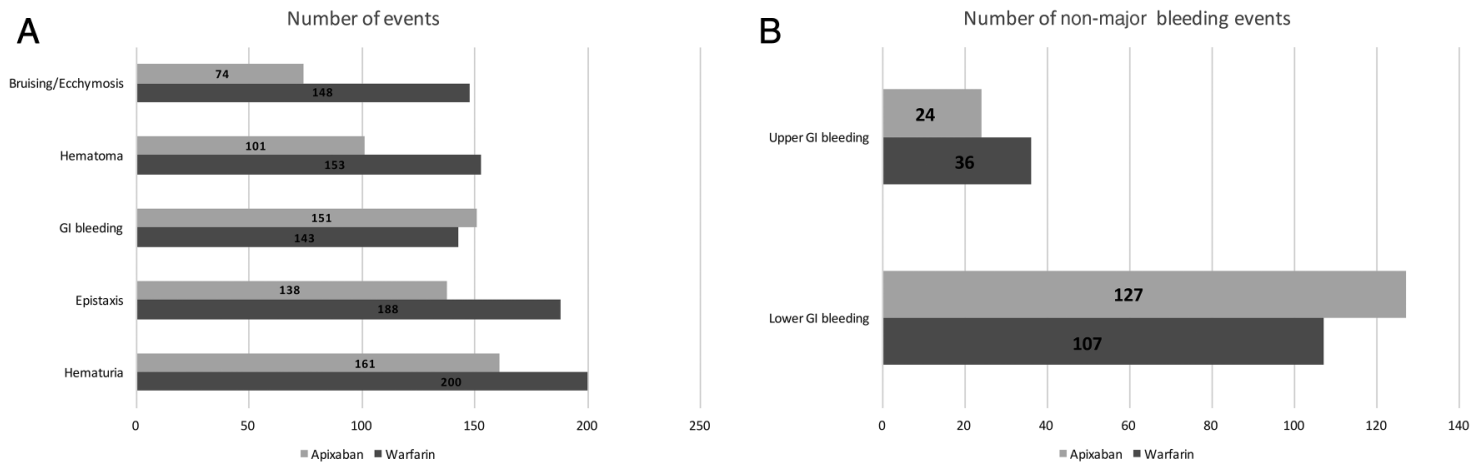

Figure 2 (A) Distribution of most common location of first non-major or minor bleeding events (1 patient with missing location; 11 patients had 2 bleeding locations reported); (B) non-major gastrointestinal bleeding location. 


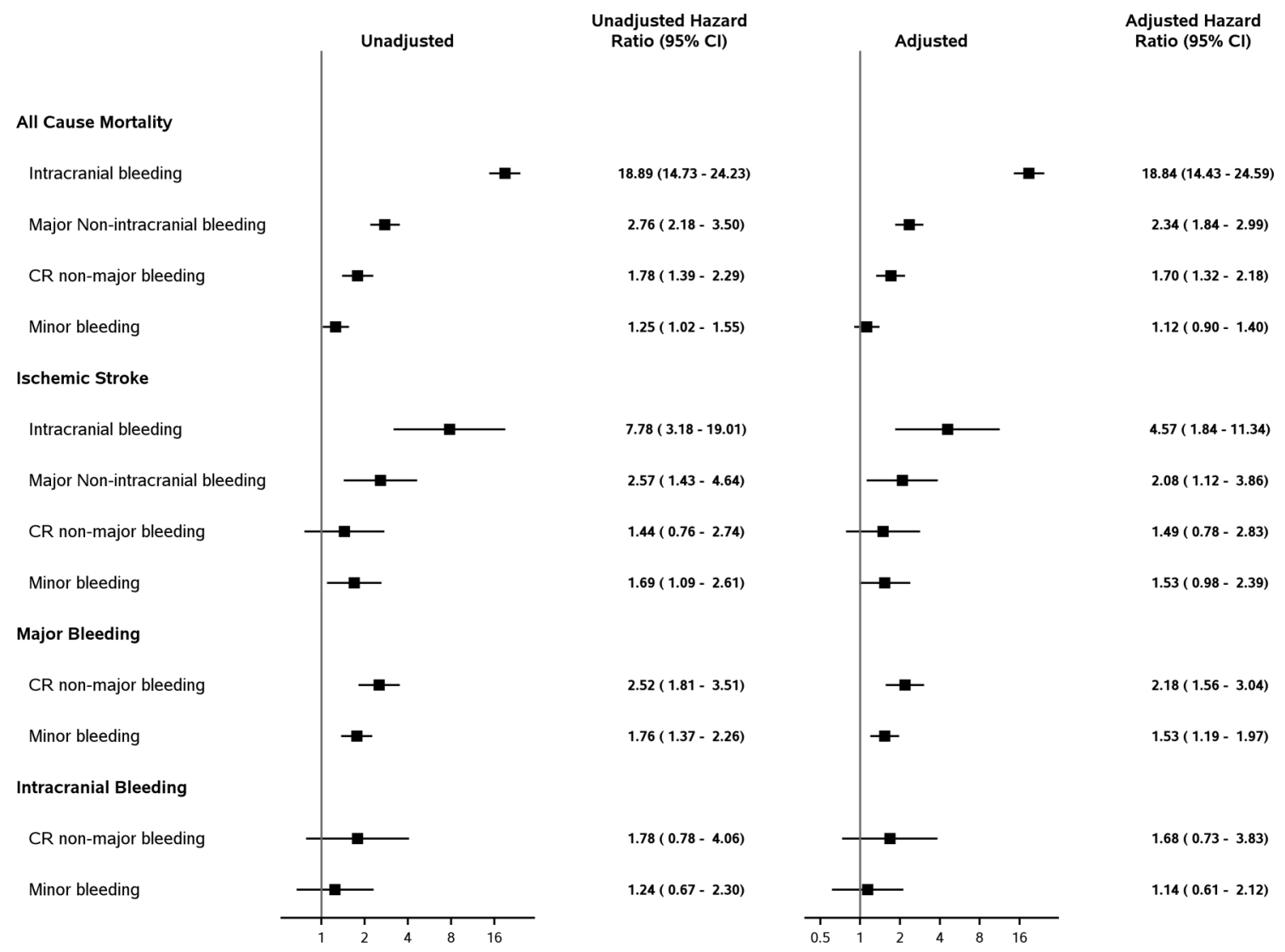

Figure 3 Effect of different severities of bleeding on subsequent outcomes (HR (95\% Cl) for yes vs no). CR, clinically relevant.

rivaroxaban and $16.1 \%$ of those treated with warfarin experienced clinically relevant non-major bleeding. Clinically relevant non-major bleeding was defined as overt bleeding not meeting the criteria for major bleeding but associated with medical intervention, unscheduled contact (visit or telephone call) with a physician, (temporary) cessation of study treatment, or associated with discomfort for the subject such as pain or impairment of activities of daily life. In the randomised evaluation of long-term anticoagulation therapy (RE-LY), ARISTOTLE and Effective Anticoagulation with Factor Xa Next Generation in Atrial Fibrillation-Thrombolysis in Myocardial Infarction 48 (ENGAGE AF-TIMI 48) trials, non-major bleeding was not part of the primary safety outcome. In RE-LY, ${ }^{4}$ patients with minor bleeding defined as all other bleeding not fulfilling major or life-threatening bleeding, accounted for 13.6\%, 14.8\% and $16.3 \%$ for dabigatran $110 \mathrm{mg}$, dabigatran $150 \mathrm{mg}$ and warfarin, respectively. In ENGAGE AF-TIMI 48, ${ }^{11}$ clinically relevant nonmajor bleeding occurred in $8.7 \%, 6.6 \%$ and $10.2 \%$ and minor bleeding in $4.1 \%, 3.5 \%$ and $4.9 \%$ of patients for high-dose edoxaban, low-dose edoxaban, and warfarin, respectively.

Even though non-major bleeding occurred more frequently, most of the published analyses concerning prognosis and management of bleeding have focused on major bleeding. ${ }^{6} 71012$ Non-major bleeding is clinically important since it is a common complication and often results in adverse consequences, including hospitalisation and cessation of effective anticoagulation that can lead to worse subsequent clinical outcomes.

In ARISTOTLE ${ }^{3}$ non-major bleeding was less frequent with apixaban than warfarin. This is consistent with the very consistent safety profile of apixaban regarding major bleeding in the trial. ${ }^{6}$ The proportions of non-major bleeding according to location were similar for apixaban-treated and warfarin-treated patients. With the exception of lower gastrointestinal bleeding, apixaban caused less bleeding than warfarin in all other locations. The increased risk of lower gastrointestinal non-major bleeding with apixaban, including lower, rectal and haemorrhoidal bleeding, was consistent with the pattern seen in a prior major bleeding analysis of patients taking oral anticoagulants. ${ }^{13}$ Similar findings have been described in the RE-LY study, ${ }^{10}$ where rates of overall gastrointestinal major bleeding were higher with both dabigatran 110 and $150 \mathrm{mg}$ compared with warfarin $(1.4 \%$, $1.9 \%$ vs $1.3 \%)$. Lower gastrointestinal major bleeding was almost twice as high with dabigatran versus warfarin ( $47 \%$ vs $25 \%)$. In ROCKET AF, ${ }^{12}$ major or clinically relevant non-major gastrointestinal bleeding, including upper, lower and rectal, was statistically significantly increased with rivaroxaban compared with warfarin $(5.5 \%$ vs $4.1 \%, p<0.001)$. Similar patterns were seen in the ENGAGE AF-TIMI 48 trial, where gastrointestinal bleeding, including both upper and lower gastrointestinal locations, was significantly more frequent in patients receiving high-dose edoxaban than warfarin $(1.51 \%$ vs $1.23 \%, \mathrm{p}=0.03) .{ }^{11}$ With increased age, the prevalence of gastrointestinal tract conditions, such as diverticulosis and angiodysplasia, ${ }^{14}$ and the risk of related bleeding from affected areas increases and when coupled with the mechanism of action of NOACs ${ }^{15}$ might help explain the association between NOACs and lower gastrointestinal bleeding.

Patients presenting with non-major bleeding who were treated with apixaban were slightly more likely to require medical or surgical consultation $(78.8 \%)$ compared with those treated with warfarin $(70.1 \%)$. The reasons for this may include the play of chance, unblinding of treatment, excessive concern about the use of a novel anticoagulant like apixaban and lack of knowledge about the management of bleeding with the new oral anticoagulants. It is important to note that the rates of bleeding events are low and that there were fewer patients with bleeding, regardless of location, in the apixaban-treated group (figure 3 ). 
As seen in patients with major bleeding in ARISTOTLE, ${ }^{7}$ warfarin treatment was more often associated with surgical intervention and/or change in antithrombotic therapy, as well as more hospitalisations than apixaban. These findings reinforce the safer profile of apixaban compared with warfarin.

The key findings of the present study are that (a) the incidence of non-major bleeding is high in this population; (b) apixaban caused significantly less non-major bleeding than warfarin; and (c) there is an independent increased risk of death and subsequent major bleeding associated with clinically relevant nonmajor bleeding, suggesting that physicians should chose the most efficacious antithrombotic strategy for stroke prevention, but also balance the risk of bleeding, even if minor.

\section{Limitations}

Patients included in ARISTOTLE represent a selected patient population that likely has a lower risk of bleeding than unselected patients in clinical practice. Thus, rates of non-major bleeding events may have been underestimated. Many of the analyses in this manuscript are observational and unmeasured confounders limit our ability to conclude a cause and effect relationship between non-major bleeding and clinical outcomes.

\section{Conclusion}

In the ARISTOTLE trial, non-major bleeding was common, associated with adverse outcomes including mortality and subsequent major bleeding and was less frequent with apixaban than warfarin. When non-major bleeding occurred, apixaban was less frequently discontinued than warfarin. These findings reinforce the safer profile of apixaban versus warfarin for patients with $\mathrm{AF}$, and highlight the importance of any severity of bleeding in patients with AF treated with anticoagulation therapy, suggesting that non-major bleeding, including minor bleeding, might not be minor.

\section{Key messages}

\section{What is already known on this subject?}

Major bleeding is a marker of worse outcomes in patients with atrial fibrillation (AF) using oral anticoagulants.

\section{What might this study add?}

In our study, non-major bleeding was common, associated with adverse outcomes including mortality, and was significantly less frequent with apixaban than warfarin.

\section{How might this impact on clinical practice?}

Our findings reinforce the safer profile of apixaban than warfarin for patients with AF and highlight the importance of any severity of bleeding in patients with AF treated with anticoagulation therapy, suggesting that non-major bleeding, including minor bleeding, might not be minor.

Contributors MCB and RDL had full access to all the data in the study and take responsibility for the integrity of the data and the accuracy of the data analysis. The authors, independent of the sponsor, are responsible for the design and conduct of the study; collection, management, analysis and interpretation of the data; and preparation, review and approval of the manuscript. The sponsor had the right to review the manuscript, but did not have any input into the decision to submit the manuscript for publication.

Funding The ARISTOTLE study was supported by Bristol-Myers Squibb, Princeton, NJ and Pfizer, New York, NY.
Competing interests MCB: Research grants from Bristol-Myers Squibb, Bayer and Boehringer Ingelheim. RDL: Research grants from Bristol-Myers Squibb,

GlaxoSmithKline; Consulting for Bayer, Boehringer Ingelheim, Bristol-Myers Squibb, GlaxoSmithKline, Merck, Pfyzer and Portola. CH: Research grants from GlaxoSmithKline, Merck, Roche, Bristol-Myers Squibb, AstraZeneca; Speakers bureau for AstraZeneca. MH: Employee of Bristol-Myers Squibb. DV: Nothing to report. EMH: Consulting fees/honoraria from Bristol-Myers Squibb, Daiichi Sankyo, Boehringer Ingelheim, Johnson \& Johnson, Pfizer and Bayer; and Research grants from Bristol-Myers Squibb. FV: Honoraria from and consulting/advisory board for Bristol-Myers Squibb and Pfizer. SG: Research grant from the Ministry of Education and Science, Sports and Culture, Japan as a Grant-in-Aid for Scientific Research in Japan (19590871, 21590911, 24390202); a grant for the Next-Generation Supercomputer Research and Development Program supported by RIKEN; Research grants from Sanofi-Aventis, Eisai and Boehringer Ingelheim; participated in consultancy or advisory board for Eisai, Sanofi-Aventis and Otsuka. JHA: Institutional research grants from Boehringer Ingelheim, Bristol-Myers Squibb, CSL Behring, Pfizer, Sanofi, Regado Biosciences, Tenax, Vivus; consulting fee/honoraria from Bristol-Myers Squibb, CSL Behring, Daiichi Sankyo, GlaxoSmithKlein, Jannsen, Pfizer, Portola, Sohmalution, Xoma. LW: Research grants from Bristol-Myers Squibb, Pfizer, AstraZeneca, Merck \& Co., Boehringer Ingelheim, GlaxoSmithKline; Consultant/ advisory board for Bristol-Myers Squibb, Pfizer, Abbott, AstraZeneca,

GlaxoSmithKline, Boehringer Ingelheim; Other from Bristol-Myers Squibb, Pfizer, Abbott, AstraZeneca, GlaxoSmithKline, Boehringer Ingelheim. CBG: Available at https://http://www.dcri.org/about-us/conflict-of-interest/COI-Granger_2015.pdf.

Ethics approval Ethics committees at all sites approved the study.

Provenance and peer review Not commissioned; externally peer reviewed.

Open Access This is an Open Access article distributed in accordance with the Creative Commons Attribution Non Commercial (CC BY-NC 4.0) license, which permits others to distribute, remix, adapt, build upon this work non-commercially, and license their derivative works on different terms, provided the original work is properly cited and the use is non-commercial. See: http://creativecommons.org/ licenses/by-nc/4.0/

\section{REFERENCES}

1 Hart RG, Pearce LA, Aguilar MI. Adjusted-dose warfarin versus aspirin for preventing stroke in patients with atrial fibrillation. Ann Intern Med 2007;147:590-2.

2 Hylek EM, Singer DE. Risk factors for intracranial hemorrhage in outpatients taking warfarin. Ann Intern Med 1994;120:897-902.

3 Granger $\mathrm{CB}$, Alexander JH, McMurray JJ, et al. Apixaban versus warfarin in patients with atrial fibrillation. N Engl J Med 2011;365:981-92.

4 Connolly SJ, Ezekowitz MD, Yusuf S, et al. Dabigatran versus warfarin in patients with atrial fibrillation. N Engl J Med 2009;361:1139-51.

5 Patel MR, Mahaffey KW, Garg J, et al. Rivaroxaban versus warfarin in nonvalvular atrial fibrillation. N Engl J Med 2011;365:883-91.

6 Hylek EM, Held C, Alexander JH, et al. Major bleeding in patients with atrial fibrillation receiving apixaban or warfarin: The ARISTOTLE trial (Apixaban for Reduction in Stroke and Other Thromboembolic Events in Atrial Fibrillation): predictors, characteristics, and clinical outcomes. J Am Coll Cardiol 2014;63:2141-7.

7 Held C, Hylek EM, Alexander JH, et al. Clinical outcomes and management associated with major bleeding in patients with atrial fibrillation treated with apixaban or warfarin: insights from the ARISTOTLE trial. Eur Heart J 2015;36:1264-72.

8 Lopes RD, Alexander JH, Al-Khatib SM, et al. Apixaban for reduction in stroke and other Thromboembolic events in atrial fibrillation (ARISTOTLE) trial: design and rationale. Am Heart J 2010;159:331-9.

9 Schulman S, Kearon C. Definition of major bleeding in clinical investigations of antihemostatic medicinal products in non-surgical patients. J Thromb Haemost 2005;3:692-4.

10 Eikelboom JW, Wallentin L, Connolly SJ, et al. Risk of bleeding with 2 doses of dabigatran compared with warfarin in older and younger patients with atrial fibrillation: an analysis of the randomized evaluation of long-term anticoagulant therapy (RE-LY) trial. Circulation 2011;123:2363-72.

11 Giugliano RP, Ruff CT, Braunwald E, et al. Edoxaban versus warfarin in patients with atrial fibrillation. N Engl J Med 2013;369:2093-104.

12 Goodman SG, Wojdyla DM, Piccini JP, et al. Factors associated with major bleeding events: insights from the ROCKET AF trial (Rivaroxaban Once-daily Oral Direct Factor Xa Inhibition Compared with Vitamin K Antagonism for Prevention of Stroke and Embolism Trial in Atrial Fibrillation). J Am Coll Cardiol 2014;63:891-900.

13 Hylek EM, Evans-Molina C, Shea C, et al. Major hemorrhage and tolerability of warfarin in the first year of therapy among elderly patients with atrial fibrillation. Circulation 2007;115:2689-96.

14 Strate LL. Lower Gl bleeding: epidemiology and diagnosis. Gastroenterol Clin North Am 2005;34:643-64.

15 Weitz J. New oral anticoagulants: a view from the laboratory. Am J Hematol 2012;87:S133-6. 